\title{
Surgical removal of rotational atherectomy device
}

\author{
Stephen D. Waterford, MD, MS, ${ }^{a}$ Umer J. Suleman, BS, ${ }^{\mathrm{b}}$ Samin Sharma, MD, ${ }^{\mathrm{b}}$ and Farzan Filsoufi, MD,
} New York, NY

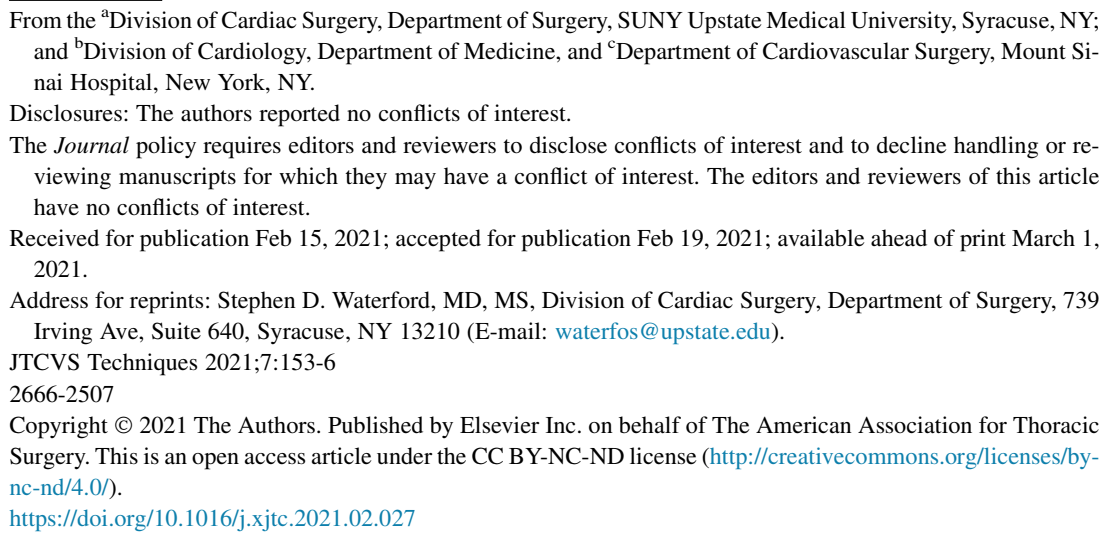

Video clip is available online.

Rotational atherectomy emerged in the 1990s and is used in percutaneous coronary intervention to manage calcified lesions. Rotational atherectomy uses a diamond-tipped burr that rotates and ablates plaque as it advances over a guidewire that is placed across a lesion. Burr entrapment is a known complication because if the burr passes beyond an incompletely destroyed plaque, it can be impossible to remove because the back of the burr cannot ablate in a retrograde fashion. Pulling vigorously on the device from outside the body can cause coronary artery dissection and rupture. There are catheter-based techniques for removal, ${ }^{1}$ including passing a second guidewire for angioplasty of the stuck segment, and use of snares. ${ }^{2}$ However, although there are many reports of catheter-based removal, there are few reports of surgical removal that include technical detail. ${ }^{3,4}$ Our goal is to review the technical details of a single case, and provide some underlying principles.

\section{CASE}

A 81-year-old woman underwent cardiac catheterization for angina due to a left anterior descending (LAD) calcified in-stent stenosis. She had undergone coronary artery bypass grafting 8 years ago, and recent catheterization showed an atretic left internal thoracic artery to LAD bypass, as well as patent vein grafts to a diagonal branch and right coronary artery. She also had prior drug-eluting stent placement to

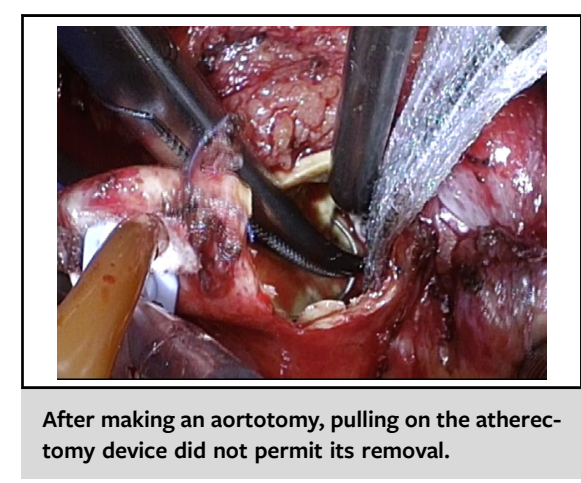

\section{CENTRAL MESSAGE \\ Rotational atherectomy device removal is not always possible by pulling back on the device through an aortotomy, and can require distal arteriotomy for removal.}

See Commentaries on pages 157 and 159.

the proximal and mid-LAD, with $40 \%$ in-stent stenosis in the proximal LAD, and $95 \%$ in-stent stenosis in the midLAD (Figure 1, $A$ ). The mid-LAD lesion was distal to the failed internal thoracic artery-LAD bypass. Because it was calcified, rotational atherectomy was performed through a femoral artery (Rotablator; Boston Scientific, Marlborough, Mass). After initial advancement of the burr through the lesion, pulling back did not permit its removal (Figure 1). Additional catheter-based techniques were used, namely introduction of a second guidewire with attempted angioplasty of the stuck segment, but the segment could not be crossed with the second guidewire due to resistance at this site. At this point, the cardiac surgical service was called. At the time of consultation, the patient was hemodynamically stable and free from chest pain. Electrocardiogram showed normal sinus rhythm with no ST changes or T wave abnormalities, and left ventriculogram suggested a normal ejection fraction with normal wall motion. She had received a loading dose of clopidogrel a few hours before the start of the catheterization and was initiated on a heparin drip. A computed tomography scan of her chest was performed to prepare for redo sternotomy, and she underwent an operation for its retrieval. Troponin-I 


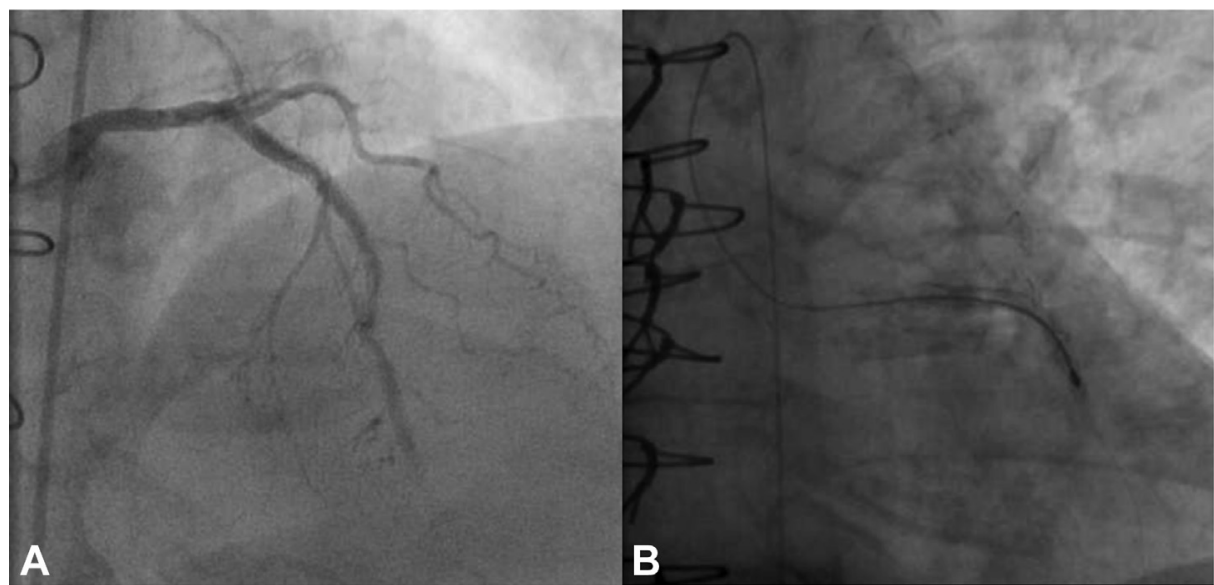

FIGURE 1. Left heart catheterization. A, Before introduction of the rotational atherectomy device, left coronary injection shows a $40 \%$ proximal left anterior descending (LAD) in-stent stenosis, 95\% mid-LAD in-stent stenosis, and distally occluded LAD. B, Final image of the rotational atherectomy device before removal, stuck in the LAD.

levels had increased from an initial postcatheterization value of $0.01 \mathrm{ng} / \mathrm{mL}$ to a final preoperative value of $5.31 \mathrm{ng} / \mathrm{mL}$ (normal reference range, $0.00-0.03 \mathrm{ng} / \mathrm{mL}$ ).

Redo sternotomy was performed and saphenous vein was harvested. A 10-cm segment of the guidewire distal to the burr was seen perforating the LAD and exiting into the pericardium (Figure 2, $A$ and $B$ ). This could not be conclusively identified on the cardiac catheterization. The aorta was clamped and antegrade and retrograde cardioplegia introduced. We attempted to remove the device in a retrograde fashion. A 2-cm transverse aortotomy was made to allow direct traction with forceps on the drive shaft proximal to the burr (Figure 2, $C$ and $D$ ). Despite considerable force, the burr could not be retrieved. Attention was then turned to the guidewire exiting the $\mathrm{LAD}$, and the $\mathrm{LAD}$ was opened longitudially at the site of the guidewire perforation. With only gentle pulling on the guidewire, the burr was easily removed distally through the coronary arteriotomy, rather than proximally through the aorta (Figure $3, A$ ). Following removal of the burr, scissors were used to divide the drive

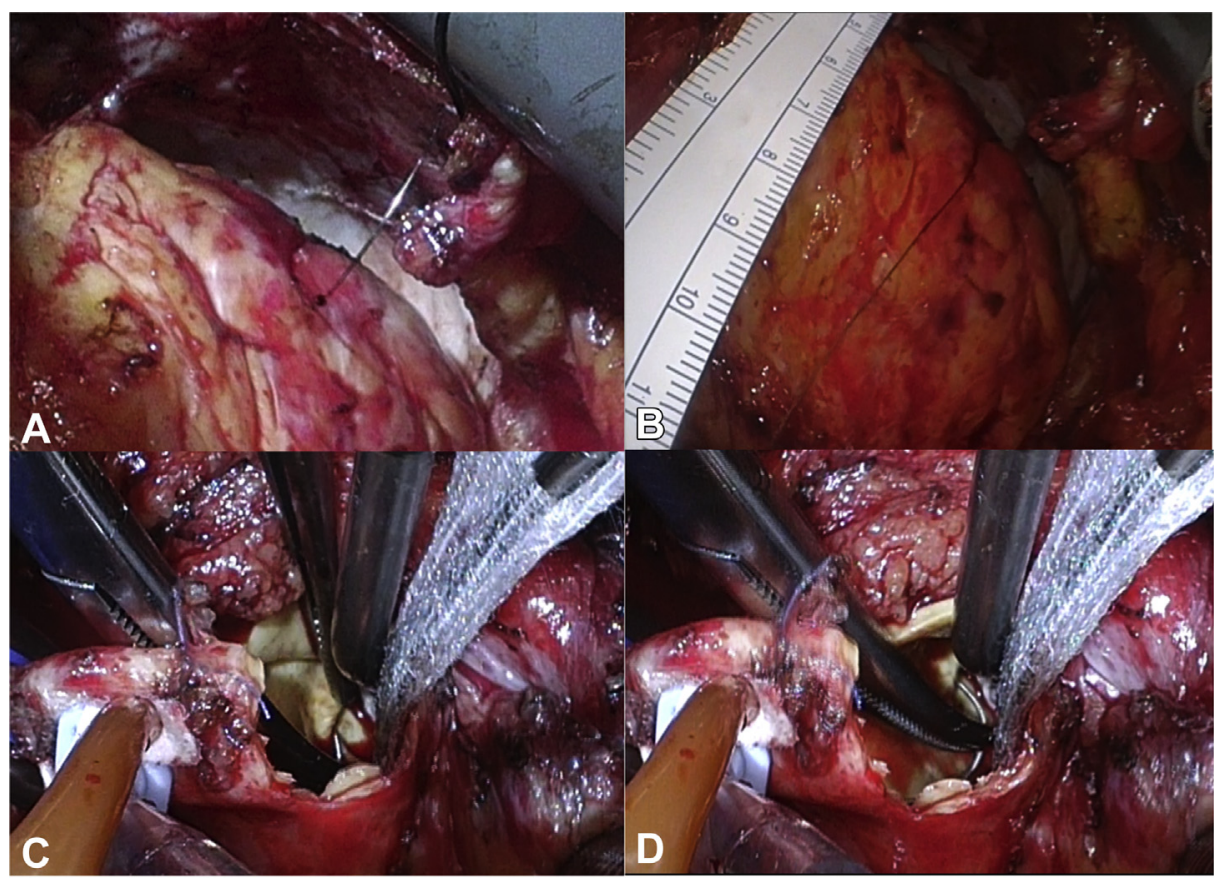

FIGURE 2. A, Guidewire is found to have perforated the left anterior descending artery. B, More than $10 \mathrm{~cm}$ of the guidewire visible in the pericardial space. C, On making an aortotomy the device is easily identified. D, Traction with a Schnidt clamp did not allow its removal. 


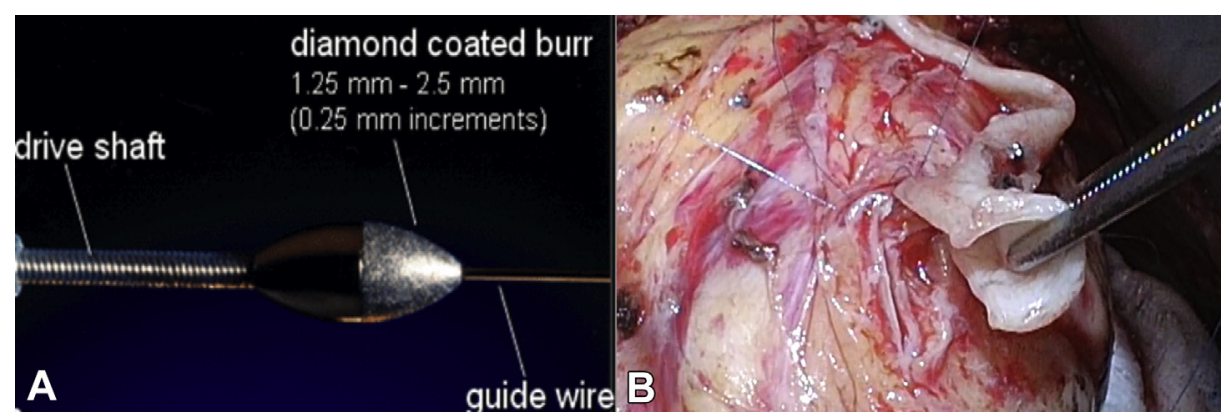

FIGURE 3. The removed atherectomy device (A) showing the $0.014^{\prime \prime}$ guidewire on the left, the burr in the center, and what is termed the helical driveshaft on the right. B, A long arteriotomy $1 \mathrm{~cm}$ in length was then made in the left anterior descending artery (LAD) and the vein graft sewn to it, taking care to tack up the flap on the proximal end of the LAD.

shaft, which is the portion of the device proximal to the burr, and the remainder of the drive shaft was then easily extracted from the aorta and trimmed at the level of the aorta. The aortotomy was closed. The LAD was then opened farther at the site of guidewire perforation, extending the arteriotomy $1 \mathrm{~cm}$ distally into nondissected territory. An anastomosis of saphenous vein was performed to the LAD, taking care to tack up the intimal flap proximally (Figure 3, B). Should this flap have rendered the LAD an unsuitable target, this segment would have been closed and opened more distally for a bypass. Cardiopulmonary bypass was weaned, and the portion of device from the right femoral artery to the ascending aorta was removed after heparin reversal. The patient made an uneventful recovery without stroke, respiratory or renal failure, and was discharged 10 days later. Predischarge echocardiogram showed an ejection fraction of $50 \%$. Consent was waived by our institutional review board for this report.

\section{COMMENT: TECHNICAL PEARLS}

Although surgical removal of a rotational atherectomy device seems simple, there are no reports with technical pearls, and there are a few helpful maneuvers. First, it is unlikely that pulling on the burr from within the aorta will remove it because this has likely already been attempted in a catheterization lab from the peripheral access site. This is consistent with the mechanism of entrapment, which involves passage of the burr past a partially treated lesion, followed by inability to remove the burr owing to a calcified lesion now proximal to the burr. Focusing on removing the burr distal to the burr is more likely to be fruitful. If the burr is removed at the level of the coronary artery, then one can transect the device and remove the remainder from the femoral artery without an aortotomy.

If the guidewire has perforated the artery, this greatly simplifies the operation because one knows where to open the artery. We recommend opening the artery at the site of guidewire perforation and proceeding as described above. If the guidewire has not perforated the artery, we recommend reviewing the catheterization images and seeing where the burr is located relative to diagonal branches, or marginal branches for a right coronary or circumflex artery. We would then open the artery distal to the burr, exteriorize the guidewire, and pull on the guidewire to remove the burr through the arteriotomy. One exception to this would be a burr that is entrapped inside a previously placed stent; that is, when the burr was used to treat an in-stent stenosis. In this case, it is possible that neither distal nor proximal traction on the guidewire will remove the burr because the burr can be stuck within the lattice of the stent. In this case, locating the burr based on the cardiac catheterization and opening at the location of the burr itself will permit it to be disentangled from the lattice of the stent and removed. ${ }^{3}$ Palpating for the stent and knowing where within the stent the burr is located will allow the artery to be opened in the correct location.

Finally, we recommend a thorough attempt to remove an entrapped rotational atherectomy device before abandoning the device in the heart. For instance, in the case discussed here, the patient's troponin began to rise significantly, indicating a myocardial infarction was underway. This occurs owing to occlusion of the artery by the device, whose tip can range from 1.25 to $2.5 \mathrm{~mm}$ in diameter (Figure 3, A).

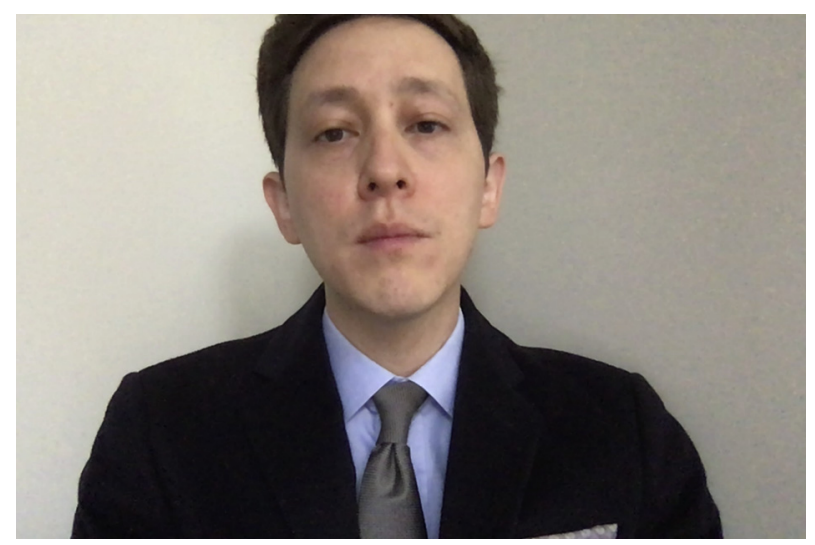

VIDEO 1. This video explains the various scenarios to prepare for during removal of a rotational atherectomy device. Video available at: https:// www.jtcvs.org/article/S2666-2507(21)00179-6/fulltext. 
In the left-sided coronary circulation, we believe it is even more important to thoroughly attempt removal because the device can occlude flow to both the LAD and left circumflex arteries, despite only being trapped in 1 of them, because the shaft passes through the left main coronary artery. If the device cannot be palpated or found after making a coronary arteriotomy, then consideration can be given to leaving it in place, although it would be unusual not to find at least the guidewire unless the arteriotomy is incorrectly positioned. Likewise, if the device is entrapped in a stent, and there is concern that removal would destroy the artery, it can be left in place. However, before abandoning extraction, we would recommend making an aortotomy and attempting proximal extraction, as well as making a coronary arteriotomy and attempting distal extraction. If the device cannot be removed with proximal and distal traction, the drive shaft still needs to be trimmed inside the aorta, and we would then perform a coronary bypass distal to the device. For the left-sided circulation, we would also give consideration to bypassing both the circumflex system and LAD.

Given the above, conduit for bypass must always be harvested for atherectomy retrieval. We recommend routinely performing a bypass distal to the site of entrapment. In many cases, the artery has been dissected by the device or by removal attempts, even if dissection was not seen on catheterization, as in the case presented. If the artery must be opened at the location of the burr, as when the burr is entrapped within a stent, we recommend closing the artery and opening it distally to perform a bypass. By contrast, if the artery can be opened distal to the burr at the location of the guidewire, a single arteriotomy technique with retrieval followed by coronary bypass is possible.

\section{CONCLUSIONS}

We have presented a case of an entrapped rotational atherectomy device and suggest various methods for its removal (Video 1).

\section{References}

1. Sullimov DS, Abdel-Wahab M, Toelg R, Kassner G, Geist V, Richardt G. Stuck Rotablator: the nightmare of rotational atherectomy. EuroIntervention. 2013;9: 251-8.

2. Chiang CH, Liu SC. Successful retrieval of an entrapped Rotablator burr by using a guideliner guiding catheter and a snare. Acta Cardiol Sin. 2017;33:96-8.

3. Fouquet O, Gomez M, Abi-Khalil W, Binuani P. Open heart surgery for a stuck Rotablator. Eur J Cardiothorac Surg. 2016;49:e149.

4. Alexiou K, Kappert U, Tugtekin SM. Entrapped coronary catheter remnants and stents: must they be surgically removed? Tex Heart Inst J. 2006;33:139-42. 